\title{
Temperatura de germinación de semillas de anón (Annona squamosa L.)
}

\section{Germination temperature of sugar apple (Annona squamosa L.) seeds}

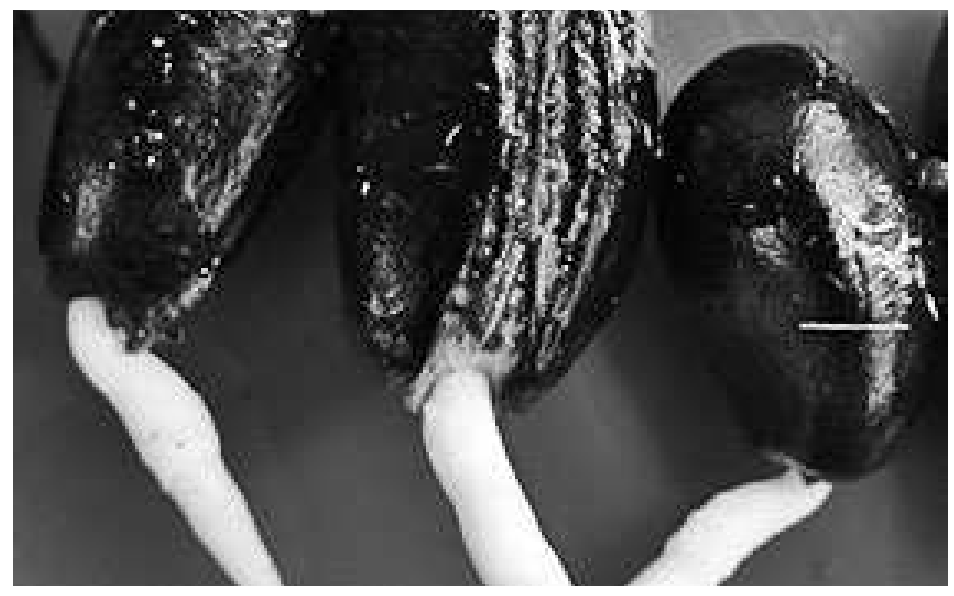

FABIO ERNESTO MARTÍNEZ M. ${ }^{1,3}$

DIEGO MIRANDA²

STANISLAV MAGNITSKIY²

Germinación de Annona squamosa L. Foto: F.E. Martínez M.

\section{RESUMEN}

La propagación sexual del anón es el método más utilizado por los productores en Colombia, sin embargo uno de los problemas del cultivo es la baja e irregular germinación de las semillas. En esta investigación se buscó establecer la temperatura óptima de germinación de semillas de A. squamosa L. Esta se realizó en el laboratorio de Fisiología Vegetal, Facultad de Agronomía, Universidad Nacional de Colombia, semillas de accesiones de Apulo (Cundinamarca) y Castilla (Tolima) fueron puestas a germinar en turba rubia húmeda sin nutrientes en un fitotrón (humedad relativa de 60\%, sin luz) durante 30 días, en dos regímenes de temperatura constante: 30 y $35^{\circ} \mathrm{C}$ y dos regímenes de temperatura alterna: $30 / 20^{\circ} \mathrm{C}$ y temperatura ambiente (media: $18,7^{\circ} \mathrm{C}$; máxima: $22,9^{\circ} \mathrm{C}$; mínima: $17,3^{\circ} \mathrm{C}$ ). Se evaluó la respuesta de las variables porcentaje de germinación (PG) cada 5 días durante 1 mes y los índices sincronía de germinación (E), tiempo medio de germinación (TMG) y velocidad media de germinación (VMG) al finalizar el ensayo. El máximo porcentaje de germinación se obtuvo a una temperatura constante de $35^{\circ} \mathrm{C}$ para las semillas provenientes de Cundinamarca como del Tolima (58\% y $57 \%$, respectivamente), igualmente a esta temperatura se observó un menor TMG (18,41 días para Cundinamarca y 17,09 días para Tolima), una mayor VMG (0,035 y 0,040 semillas germinadas/día para Cundinamarca y Tolima, respectivamente) y un índice de sincronización más bajo $(0,29$ y 0,33 para Cundinamarca y Tolima, respectivamente), lo que sugiere como temperatura óptima $35^{\circ} \mathrm{C}$ para la germinación de las semillas de $A$. squamosa.

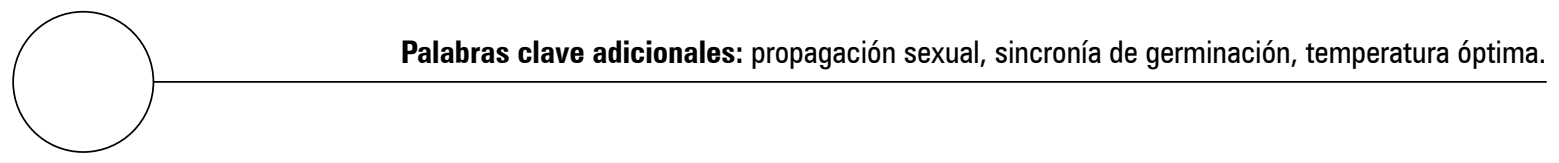

\footnotetext{
Facultad de Agronomía, Programa de Maestría en Ciencias Agrarias, Línea Fisiología de Cultivos, Universidad Nacional de Colombia, Bogotá (Colombia).

2 Facultad de Agronomía, Departamento de Agronomía, Universidad Nacional de Colombia, Bogotá (Colombia).

3 Autor para correspondencia.femartinezma@gmail.com
} 


\section{ABSTRACT}

Sexual propagation of the sugar apple is the most used type by producers in Colombia; however, one of the principal crop problems is low and irregular seed germination. This research aimed to establish the optimum germination temperature for seeds of Annona squamosa L. In the Laboratory of Plant Physiology, Faculty of Agronomy, Universidad Nacional de Colombia, seeds from Apulo (Cundinamarca) and Castilla (Tolima) accessions were planted in peat substrate trays without nutrients and subsequently placed in a phytotron chamber (relative humidity of $60 \%$, without light) for 30 days with two constant temperature regimes: 30 and $35^{\circ} \mathrm{C}$ and two alternating temperature regimes: $30 / 20^{\circ} \mathrm{C}$ and room temperature (mean: $18.7^{\circ} \mathrm{C}$, max: $22.9^{\circ} \mathrm{C}$, min: $17.3^{\circ} \mathrm{C}$ ). The response of germination percentage (GP), every 5 days for 30 days, synchronization index (E), mean germination time (MGT) and mean velocity of germination (MVG) were tested. The maximum germination rate was obtained at a constant temperature of $35^{\circ} \mathrm{C}$ with seeds from Cundinamarca and Tolima (58\% and 57\%, respectively), at this temperature, a lower MGT (18.41 days in Cundinamarca and 17.09 days in Tolima), greater MVG (0.035 and 0.040 germinated seeds/day in Cundinamarca and Tolima, respectively) and lower synchronization index (0.29 and 0.33 in Cundinamarca and Tolima, respectively) were seen, suggesting that $35^{\circ} \mathrm{C}$ is the optimum germination temperature for $A$. squamosa seeds.

Additional key words: sexual propagation, synchronization index, optimum temperature.

Fecha de recepción: 03-09-2012

Aprobado para publicación: 28-11-2012
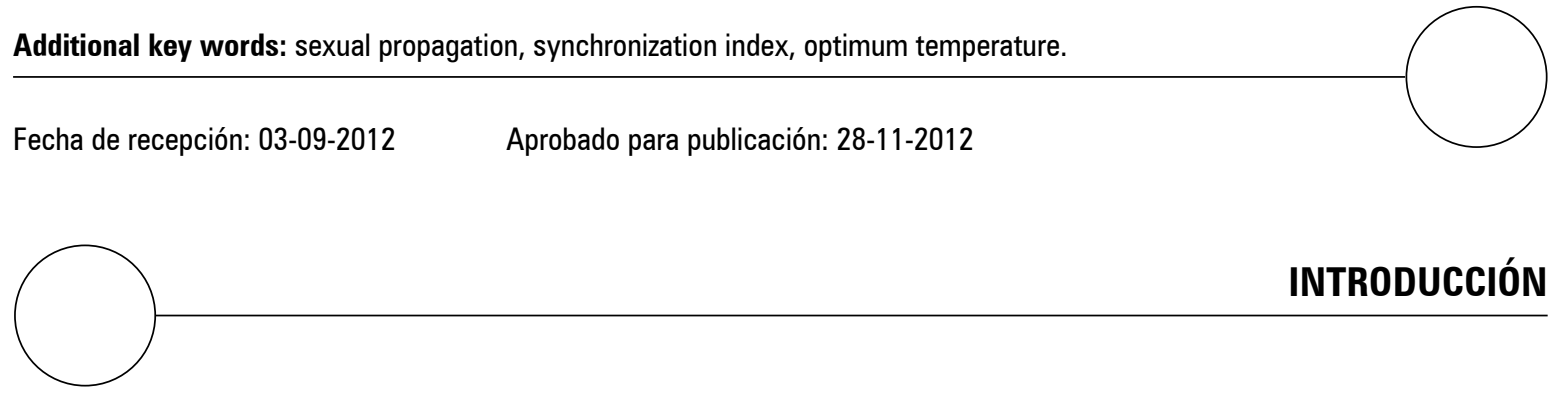

INTRODUCCIÓN

La familia Annonaceae incluye aproximadamente 50 géneros. Tres géneros (Almona, Rollinia y Asimina) producen frutos comestibles (George y Nissen, 1993). Dos géneros son de importancia comercial; Annona, que comprende unas 100 especies, y Rollinia, que incluye cerca de 50 especies (George, 1985). Las principales especies comerciales son la chirimoya (A. cherimola Mill.), el híbrido atemoya (A. cherimola Mill. x A. squamosa L.), el anón (A. squamosa L.) y la guanábana (A. muricata L.). La chirimoya es nativa de las tierras altas subtropicales de Perú y Ecuador, y se cultiva comercialmente en Chile, España, California y Nueva Zelanda (George y Nissen, 1993). La atemoya es un híbrido y al parecer un intermedio en las características morfológicas y necesidades climáticas de sus padres. Se cultiva carencialmente en Florida (EE.UU.) y Australia (George y Nissen, 1987). La guanábana se distribuye en regiones tropicales de América Central y este de India (Popenoe, 1974).
El anón es la especie más ampliamente distribuida del género Annona en el mundo. Se cultiva en la zona tropical de Suramérica, en el sur de México, el occidente de la India, Bahamas, Bermudas, y en el sur de la Florida. Igualmente, en las zonas cálidas tropicales de América, Jamaica, Puerto Rico, Barbados, el sur de la India y en las regiones secas del norte de Queensland (Australia) se encuentra en forma silvestre en praderas $y$ bosques (Morton, 1987; Hoyos, 1989). El cultivo es extenso en India, mientras que en Brasil es uno de los frutos más importantes, siendo llamativo en los mercados de Bahía. En Colombia, se encuentra en la Costa Atlántica y en las zonas secas de los valles interandinos, en los departamentos de Valle, Caldas, Huila, Tolima, Cundinamarca, Meta y los Santanderes, entre los 450 y 1.500 msnm (Lotero, 1976). A pesar de que aún no se exporta oficialmente anón, la opinión de expertos en frutas promisorias lo clasifican como fruto de alto potencial de mercado 
en el exterior. En el mercado nacional, el anón es de alta demanda y alto precio, bordeando los $\$ 4.000$ por kg. Esto explica el amplio margen de intervención en prácticas adecuadas de mercadeo, lo cual, permite mejorar significativamente el precio al productor. Por otro lado, el anón presenta posibilidades reales en el mercado internacional, prueba de ello es el interés de la Corporación Colombia Internacional y exportadoras como "El Tesoro Fruit", quien ha enviado las primeras muestras a Alemania (Martínez, 2012).

En los taxa cultivados de Annona, autores como Pinto (2005a) y Padilla y Encina (2003) han indicado problemas en la propagación sexual, principalmente la germinación irregular, la cual, es debida a diferentes tipos de latencia (Ferreira et al., 1997; Moreno et al., 1999; Pinto, 1975; Lobo et al., 2007), atribuidos, entre otros factores, al grado de madurez de la semilla, la cual está determinada por factores climáticos, en lugar de ser innata (Pinto, 2005b).

Según Bewley y Black (1994) la temperatura puede afectar la absorción de agua y las reacciones bioquímicas que regulan el metabolismo involucrado en el proceso de germinación. Las semillas presentan una capacidad germinativa, en límites bien definidos de temperatura característicos de cada especie. Por tanto, es de gran interés fisiológico determinar las temperaturas mínima, óptima y máxima de germinación. En la temperatura óptima se propicia un máximo porcentaje de germinación en menor espacio de tiempo (Mayer y Poljakoff-Mayber, 1989). Las temperaturas máximas aumentan la velocidad de germinación pero solamente las semillas más vigorosas germinan, generando así un bajo porcentaje de germinación. Por su parte, las temperaturas mínimas reducen la velocidad de germinación y alteran la uniformidad de emergencia (Carvalho y Nakagawa, 2000).

Con relación a la temperatura de germinación en A. squamosa hay pocos reportes y los existentes son controversiales. Varios autores como Zuca- reli et al. (2007), Ferreira et al. (1997), Ba et al. (2012) y Kavati (1992) coinciden en temperaturas de $30^{\circ} \mathrm{C}$ como la más adecuada para esta especie, aunque también se cita la temperatura alterna $20 / 30^{\circ} \mathrm{C}$. Por tanto, el objetivo de este trabajo fue determinar la temperatura de germinación más adecuada para las semillas de la especie A. squamosa cultivada en dos zonas de potencial productivo para la especie en Colombia.

\section{METODOLOGÍA}

Esta investigación fue realizada en el laboratorio de Fisiología Vegetal y en el laboratorio de Recursos Genéticos de la Facultad de Agronomía, Universidad Nacional de Colombia, Bogotá.

\section{Selección de material vegetal}

El material seleccionado se obtuvo de las accesiones colectadas para el desarrollo del proyecto del Banco Colombiano de Germoplasma de Anón que forma parte del programa: Material de Siembra y Mejoramiento Genético del Anón (Annona squamosa L.). Durante el desarrollo del proyecto se realizaron expediciones de colecta de material en todo el país. La selección de los materiales a emplear en el estudio se realizó buscando accesiones contrastantes, basado en los valores de descriptores de caracterización morfo-agronómica tomados en campo y en laboratorio, así como a la tradición en la producción de la especie. De todas las zonas visitadas se seleccionaron las accesiones C2AS224, C2AS225 y C2AS226 (C: Cundinamarca; 2: código de municipio; AS: especie Annona squamosa; 224: número de consecutivo) colectadas en el municipio de Apulo, Cundinamarca y las accesiones T7AS181 y T7AS181 de Castilla (Tolima) debido a que los individuos se encuentran distribuidos en agroecosistemas y no en solares o de forma espontánea en jardines. En particular se destaca la potencialidad frutícola del municipio de Apulo como principal productor nacional de anón, que se debe a la calidad intrínseca que presenta el fruto (alto contenido 
de azúcares) en el ecosistema bosque seco tropical (Martínez, 2012).

\section{Obtención de semillas y manejo del sustrato}

Las semillas se obtuvieron a partir de frutos maduros y blandos al tacto, se lavaron con agua a temperatura ambiente para remover hongos, suelo o cualquier residuo presente en su superficie. Luego se separó la corteza (pericarpio) de la pulpa (mesocarpio) y se retiró manualmente la semilla de la pulpa y el arilo; posteriormente se lavaron y se colocaron en toallas de papel absorbente durante $3 \mathrm{~d}$ a temperatura ambiente con el fin de secarlas. Las semillas se desinfectaron con inmersión en hipoclorito de sodio al 1\% durante 9 min, lavado con agua destilada y etanol al $96 \%$, finalmente, se realizó una limpieza con agua destilada para retirar en etanol que pudiera quedar en la semilla.

El sustrato de germinación fue turba rubia $\left(\right.$ Klassmann $\left.{ }^{\circledR}\right)$. Con el fin de evitar la presencia de hongos o bacterias se desinfectó tanto la turba como las semillas usadas en el desarrollo del ensayo. La desinfección de la turba se realizó con Benomyl $\left(1 \mathrm{~g} \mathrm{~L}^{-1}\right)$ y Vitavax $\left(2 \mathrm{~g} \mathrm{~L}^{-1}\right)$.

\section{Temperatura de germinación}

Se evaluó la germinación de las semillas en cuatro regímenes de temperatura: $30^{\circ} \mathrm{C}, 30 / 20^{\circ} \mathrm{C}(12$ h), $35^{\circ} \mathrm{C}$ y temperatura ambiente (media: $18,7^{\circ} \mathrm{C}$; máxima: $22,9^{\circ} \mathrm{C}$; mínima: $17,3^{\circ} \mathrm{C}$ ) para la cual las semillas fueron puestas en bandejas con turba Klassmann ${ }^{\circledR}$ como sustrato sin nutrientes como sustrato, a una profundidad equivalente al doble de su longitud y luego puestas en un fitotrón Conviron CMP3244 (Winnipeg, Canadá), con el régimen de temperatura requerido en una humedad relativa de $60 \%$ y en ausencia total de luz, ya que las semillas de $A$. squamosa son indiferentes a las condiciones de luz (Ferreira et al., 1997).

Se estableció un diseño factorial completamente al azar con cinco repeticiones por tratamiento.
Se realizaron observaciones de germinación cada $5 \mathrm{~d}$ durante $30 \mathrm{~d}$, ya que las semillas que no germinan después de este periodo pueden considerarse latentes (Baskin y Baskin, 2001) o ya no son viables. Se registraron como semillas germinadas aquellas en las que hubo protrusión de la radícula y esta fue mayor a $3 \mathrm{~mm}$.

Se determinó la humedad y viabilidad de las semillas antes de establecer el ensayo, encontrándose para las accesiones T7AS181 y T7AS181 de Castilla (Tolima) una humedad de 12,2\% y $11,9 \%$ y un porcentaje de viabilidad de $82 \%$ y 78\%; para las accesiones C2AS224, C2AS225 y C2AS226 de Apulo, Cundinamarca, una humedad de $10,7 \%, 11,3 \%$ y $10,4 \%$ y porcentajes de viabilidad de $81 \%, 76 \%$ y $87 \%$, respectivamente.

\section{Análisis de datos}

Con los datos del muestreo en el tiempo se calculó el porcentaje de germinación (PG), índice de sincronía (E), tiempo medio de germinación (TMG) y velocidad media de germinación (VMG) siguiendo las ecuaciones presentadas en la tabla 1.

Se evaluaron los supuestos de normalidad y homogeneidad de varianzas, los datos que no presentaron normalidad en los residuales fueron transformados con la función arcosen $\sqrt{ }(\mathrm{PG} / 100)$, usualmente utilizada en estudios de germinación (Wagner et al., 2006). Se realizó un Anava y se llevó a cabo la prueba de Tukey $(P \leq 0,05)$. Se usó el paquete estadístico SAS (Statistical Analysis System), versión 9.1.

\section{Ajustes a modelos de regresión}

La respuesta del PG a la temperatura para ambas localidades fue ajustada a un modelo logístico tal y como se define a continuación:

$y=\frac{K}{1+B e^{(-A t)}}$ 
Tabla 1. Fórmulas empleadas para el cálculo de variables de germinación. Tomado de Ranal y Santana (2006).

\begin{tabular}{|l|r|r|r|}
\hline Variable & Ecuación & Unidad & Ecuación No. \\
\hline PG & $\left(\frac{\mathrm{N}}{\mathrm{N}_{\mathrm{s}}}\right)^{*} 100$ & $\%$ & (1) \\
\hline VMG & $\sum_{i=1}^{k} n_{i} l_{i}$ & Semillas germinadas/día & (2) \\
\hline TMG & $\sum_{t=1}^{k} n_{i} t_{i} / \sum_{t=1}^{k} n i$ & Días & (3) \\
\hline $\mathrm{E}$ & $-\sum_{i=1}^{k} f_{i} \log _{2} f_{i}$ & Adimensional & (4) \\
\hline
\end{tabular}

ni = número de semillas germinadas en la iésima toma de datos.

ti $=$ tiempo (en días) de la iésima toma de datos.

$K=$ tiempo (en días) de duración de la prueba de germinación.

$\mathrm{N}=$ número de semillas germinadas.

$\mathrm{N}_{\mathrm{s}}=$ número de semillas totales.

$\mathrm{f}_{\mathrm{i}}=$ frecuencia relativa de germinación

En donde $K$ es el máximo valor que alcanza la variable, en este caso el peso de las semillas (g) o el porcentaje de germinación (\%), A es el producto entre el valor inicial "a" por el máximo " $K$ ", B es un parámetro de escala sobre el tiempo $t$ que influencia la tasa de crecimiento.

\section{RESULTADOS}

Para las semillas de las dos localidades hubo efectos significativos $(P \leq 0,001)$ de la temperatura sobre el porcentaje de germinación (PG), la velocidad de germinación (VMG), el tiempo medio de germinación (TMG) y el índice de sincronía (E). La localidad y la interacción entre localidad y temperatura no tuvieron efectos sobre las variables (tabla 2).

El máximo porcentaje de germinación se obtuvo a una temperatura constante de $35^{\circ} \mathrm{C}$ después de $30 \mathrm{~d}$ a partir de la siembra, para Cundinamarca y para Tolima (58\% y 57\%, respectivamente).
En general el PG fue drásticamente reducido a la temperatura de $30^{\circ} \mathrm{C}$ y $30 / 20^{\circ} \mathrm{C}$ y fue completamente nulo a temperatura ambiente (figura 1A). Las temperaturas alternas ofrecen una mejor germinación que las temperaturas constantes según lo expuesto por Hartmann et al. (1997), sin embargo en este estudio las temperaturas alternas tuvieron un efecto negativo sobre la expresión de la germinación.

Para las semillas provenientes de las dos localidades, el tiempo medio de germinación (TMG) no fue estadísticamente diferente entre las temperaturas de 35,30 y $30^{\circ} \mathrm{C} / 20^{\circ} \mathrm{C}$, pero sí con la temperatura ambiente, esto debido a que no se reportó germinación a los 30 d de evaluación. Sin embargo, se observó un menor TMG en las semillas provenientes de las dos localidades incubadas a una temperatura de $35^{\circ} \mathrm{C}(18,41 \mathrm{~d}$ para Cundinamarca y 17,09 d para Tolima) (figura 1B).

Una situación similar se observó para la VMG. A temperatura ambiente las semillas presen- 
Tabla 2. Significancia de la prueba $F$ para el régimen de temperaturas en el porcentaje de germinación (PG), tiempo medio de germinación (TMG), velocidad media de germinación (VMG) y sincronización (E) en semillas de A. squamosa incubadas durante $30 \mathrm{~d}$ en turba húmeda.

\begin{tabular}{|l|c|c|c|c|} 
& PG & TMG & VMG & N \\
\hline Localidad (LOC) & NS & NS & NS & NS \\
\hline Temperatura (TEMP) & $* * *$ & $* * *$ & $* * *$ & NS \\
\hline TEMP X LOC & NS & NS & NS & N \\
\hline
\end{tabular}

${ }^{* * *}=P \leq 0,001,{ }^{* *}=P \leq 0,01,{ }^{*}=P \leq 0,05, \mathrm{NS}=$ no significativo

tan una VMG estadísticamente menor, 0,035 y 0,040 semillas/día para Apulo y Castilla, respectivamente, en comparación con las semillas incubadas en las demás temperaturas evaluadas, sin embargo, pese a que no hubo diferencias estadísticas entre 35,30 y $30 / 20^{\circ} \mathrm{C}$, es posible apreciar cómo las semillas incubadas a $35^{\circ} \mathrm{C}$ presentan una VMG más alta, 0,055 y 0,059 semillas/día para Apulo y Castilla, respectivamente (figura 1C). Para las dos localidades el índice de sincronización más bajo se obtuvo cuando las semillas se incubaron a $35^{\circ} \mathrm{C}$ durante 30 d, 0,29 y 0,33 para Apulo y Castilla, respectivamente, lo que indica que la germinación a esta temperatura fue más sincronizada en comparación con las temperaturas de 30 y $30 / 20^{\circ} \mathrm{C}$, pues bajos valores en este valor indican una mayor sincronización en la germinación (Ranal y Santana, 2006; figura 1D).

En las figuras 2 y 3 se presentan los resultados obtenidos con el análisis de regresión de los PG. Para las semillas obtenidas de Apulo, Cundinamarca, la función logística fue la que mejor describió el patrón de germinación en los tres regímenes de temperatura. A $35^{\circ} \mathrm{C}$ los valores del PG fueron mucho mayores a los obtenidos a $30 \mathrm{y}$ $30 / 20^{\circ} \mathrm{C}$. Durante los primeros 20 d se presentó un incremento acelerado del PG (máximo valor $K=69,36)$ tendiendo a la estabilización a los 30 $\mathrm{d}$ de incubación.

Para la localidad de Castilla, Tolima, el modelo logístico fue el que mejor describió el patrón de germinación para todas las temperaturas. El comportamiento de la curva logística para semi- llas germinando a $35^{\circ} \mathrm{C}$, en la localidad del Tolima, indica que en los primeros $15 \mathrm{~d}$ se presentó un incremento acelerado del PG el cual llega a su máximo valor $(58,2 \%)$ y se estabiliza luego a los 30 dds. En las dos localidades pueden apreciarse valores más altos en el PG cuando las semillas fueron incubadas a $35^{\circ} \mathrm{C}$, durante los $30 \mathrm{~d}$ de evaluación. Estos resultados coinciden con los expresados para PG, VMG, TMG y E, indicando que la temperatura de $35^{\circ} \mathrm{C}$ es la que permite una mejor expresión de la germinación. En contraste, las temperaturas de 30 y $30 / 20^{\circ} \mathrm{C}$, tienen poco efecto sobre el potencial germinativo de la especie.

Promedios con letras distintas indican diferencia significativa según la prueba de Tukey en cada día de aplicación $(P \leq 0,01)$.

Estos resultados difieren de los encontrados por diferentes autores (Zucareli et al., 2007; Ferreira et al., 1997; Ba et al., 2012) en estudios de germinación en $A$. squamosa, y en otras especies. Los autores reportan que los mayores PG y VMG, así como menores TMG, se obtienen a una temperatura de $30^{\circ} \mathrm{C}$ y temperaturas alternas $20 / 30^{\circ} \mathrm{C}$. Indican también que en temperaturas menores a $23^{\circ} \mathrm{C}\left(20\right.$ y $\left.17^{\circ} \mathrm{C}\right)$ y mayores a $30^{\circ} \mathrm{C}\left(33\right.$ y $\left.42^{\circ} \mathrm{C}\right)$ causan los valores más bajos en estas variables, limitando el potencial de germinación. Los autores concluyen que las semillas de $A$. squamosa son indiferentes a las condiciones de luz y que las temperaturas elevadas favorecen el proceso germinativo, lo cual es corroborado por Kavati (1992), quien argumenta que esta especie es de clima cálido. 
También en otras especies de Annonaceae, Oliveira et al. (2005) en $A$. montana y Nepomuceno Costa et al. (2011) en A. muricata y A. emarginata reportaron que la temperatura de germinación más adecuada es $30^{\circ} \mathrm{C}$, ya que se obtienen los valores más altos de PG (no mayores al 61\%) y VMG, mientras que temperaturas mayores $\mathrm{O}$ menores $\left(35,20\right.$ y $\left.17^{\circ} \mathrm{C}\right)$ la germinabilidad disminuye o no se presenta germinación. En otras especies como A. cherimola (Toll-Jubes et al.,
1975), A. senegalensis (Ba et al., 2012) el mejor PG (55\%) fue obtenido a $25^{\circ} \mathrm{C}$.

En especies como Cecropia glaziovi (Godoi y Takaki, 2004) el mayor PG y VMG se obtiene a una temperatura de $30^{\circ} \mathrm{C}$, mientras que en Albizia lebbeck, Gliricidia sepium y Bauhinia purpurea los mayores porcentajes de germinación final se obtuvieron a temperatura alterna de $25 / 35^{\circ} \mathrm{C}$ (Reino et al., 2008).

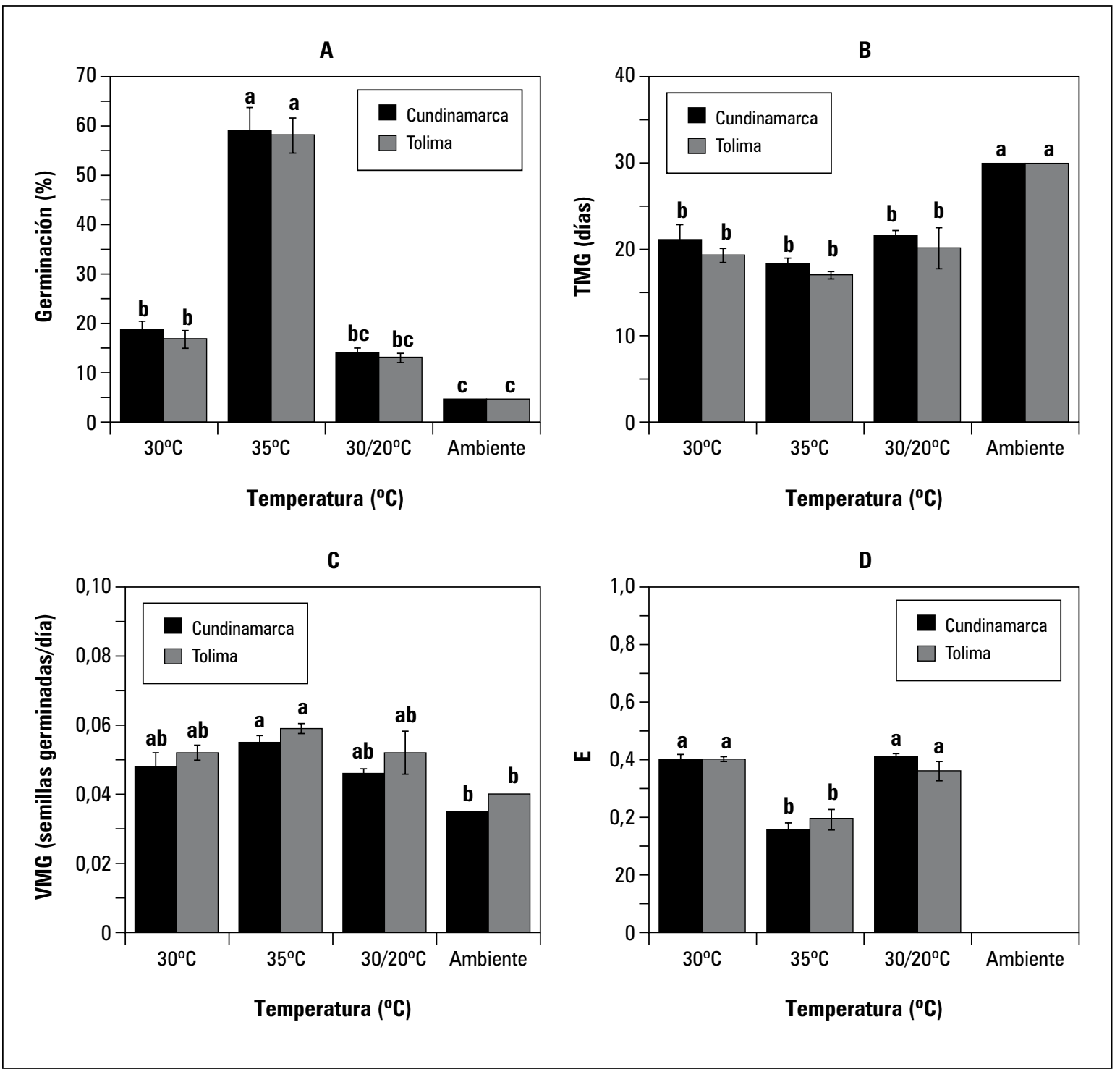

Figura 1. Efecto de las temperaturas sobre: A. Porcentaje de germinación (PG), B. Tiempo medio de germinación (TMG), C. Velocidad media de germinación (VMG) y D. Índice de sincronía (E) en semillas de A. squamosa incubadas durante $\mathbf{3 0} \mathbf{d}$ en turba húmeda. Promedios con letras distintas en cada serie indican diferencia significativa según la prueba de Tukey $(P \leq 0,05)$. 


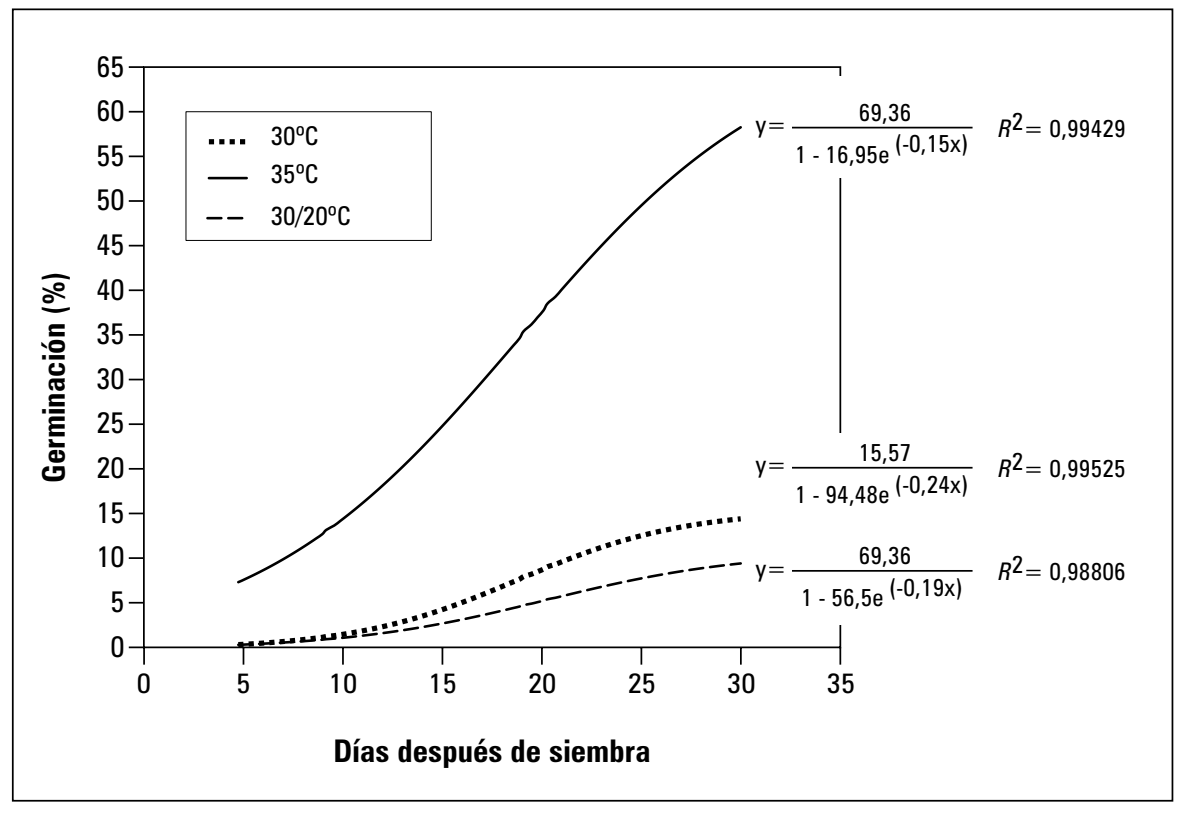

Figura 2. Porcentajes de germinación (PG) acumulada (PG) de semillas provenientes de Apulo (Cundinamarca), incubadas durante $30 \mathrm{~d}$ en turba húmeda bajo tres regímenes de temperatura.

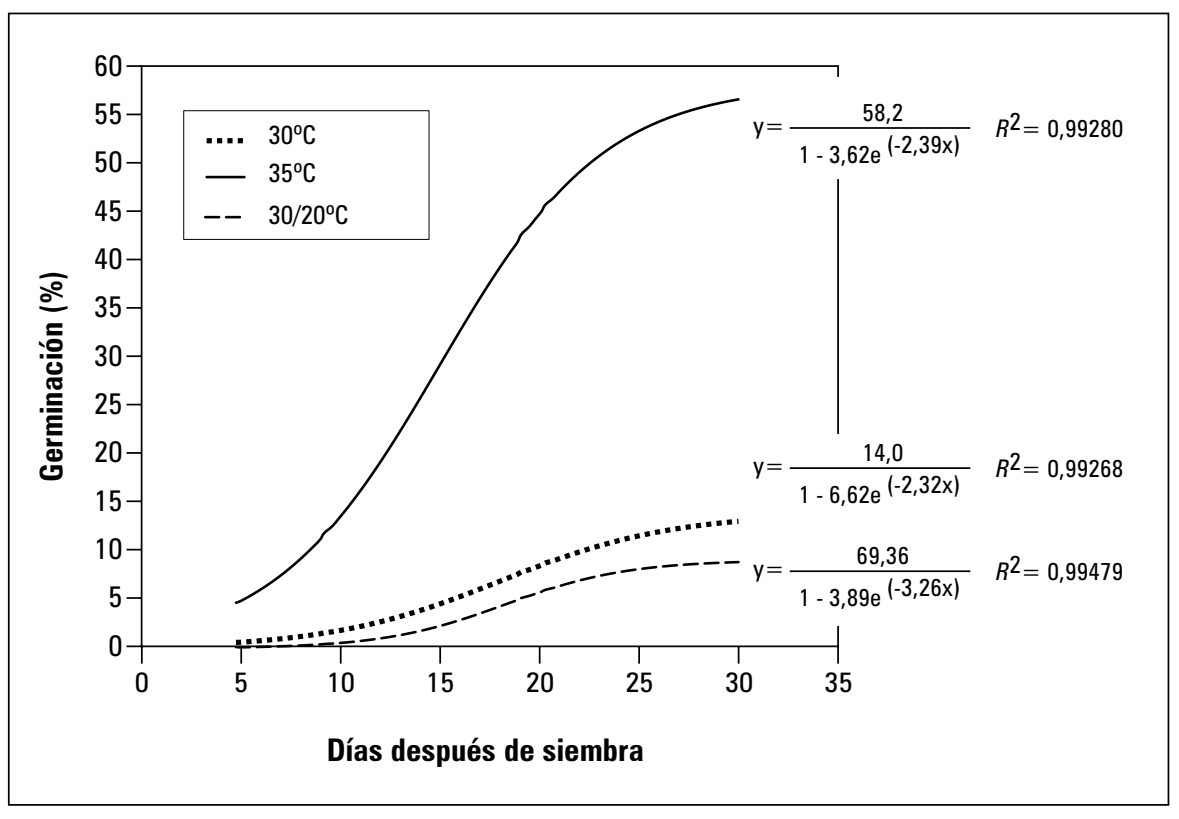

Figura 3. Porcentajes de germinación acumulada (PG) de semillas provenientes de Castilla (Tolima), incubadas durante 30 d en turba húmeda bajo tres regímenes de temperatura. 
La temperatura afecta la germinación y la tasa de germinación final varía con la temperatura (Come, 1982). Borges y Rena (1993) postulan que la temperatura de 20 a $30^{\circ} \mathrm{C}$ es adecuada para la germinación de semillas de la mayoría de las especies tropicales. Sin embargo, en un estudio realizado por Cavalcante y Pérez (1995), las especies tropicales presentan una notable tolerancia a altas temperaturas, mostrando generalmente un límite máximo $\leq 35^{\circ} \mathrm{C}$, en tanto estas son sensibles a temperaturas bajas, presentando generalmente un límite inferior $>5^{\circ} \mathrm{C}$. En el presente estudio los mejores valores obtenidos para todas las variables evaluadas (PG, TMG, VMG y E) fueron obtenidos a una temperatura constante de $35^{\circ} \mathrm{C}$ pudiéndose considerar esta, como la temperatura óptima de germinación, si tenemos en cuenta que Mayer y Poljakoff-Mayber (1989) consideran que la temperatura óptima de germinación es aquella en la que se obtiene el más alto porcentaje de germinación en el menor tiempo.

Castro (2003) obtuvo resultados similares estudiando semillas de Tabebuia capitata. El autor verifica que con las temperaturas de 30 y $35^{\circ} \mathrm{C}$ se obtienen los mayores porcentajes de germinación, emergencia y para formación de plántulas normales. Sin embargo, nuestros resultados difieren de los encontrados por otros autores en la misma especie y con especies relacionadas en diferentes regiones del mundo, en donde hay un mejor comportamiento de las variables de germinación en temperaturas no mayores a $30^{\circ} \mathrm{C}$. Esto puede deberse a que a esta temperatura la permeabilidad de la testa al agua es mayor que a $30^{\circ} \mathrm{C}$ según lo observado en la curva de imbibición para las dos localidades. De acuerdo con Tybirk (1991), las altas temperaturas contribuyen al ablandamiento de la cubierta seminal y aseguran una mejor permeabilidad de esta al agua y el aire. Adicionalmente, las temperaturas óptimas de germinación varían de una especie a otra (Ba et al., 2012) o incluso entre semillas de la misma especie debido a la región de donde fueron obtenidas (Harty y Butler, 1975).
En el presente estudio las temperaturas menores a $35^{\circ} \mathrm{C}$ : 30 y $20 / 30^{\circ} \mathrm{C}$ y temperatura ambiente presentaron valores bajos de PG, VMG y más altos de TMG y E en A. squamosa Según Carvalho y Nakagawa (2000) temperaturas por debajo la óptima tienden a reducir la velocidad del proceso germinativo, exponiendo las plántulas por mayor periodo de tiempo a factores adversos, pudiendo llevar a la reducción total de la germinación. Comparaciones entre diferentes estudios en temperaturas fluctuantes muestran que la germinación se dificulta debido a diversos factores como la presencia de temperaturas máximas y mínimas, y su duración y la tasa de calentamiento y enfriamiento de las semillas debido a estas fluctuaciones (Bewley y Black, 1982).

Tanto en Apulo como en Castilla la temperatura fue un factor limitante para la germinación de las semillas de A. squamosa ya, que las temperaturas en ambas regiones no superan los $30^{\circ} \mathrm{C}$. El comportamiento de las semillas que no germinan, puede deberse a que las condiciones de germinación no son efectivas para desbloquear la latencia primaria (Bewley y Black, 1994). Los resultados muestran que las semillas de ambas localidades son dependientes de la temperatura para su germinación. De acuerdo con Armitage (1994), la temperatura es el factor ambiental más importante en el control de la germinación debido a sus efectos sobre los cambios en la membrana plasmática y mitocondria así como en la desnaturalización de proteínas (Van Vuuren y Lubbe, 2000).

\section{CONCLUSIONES}

Las semillas de anón de ambas localidades son dependientes de la temperatura para su germinación. Los mejores valores obtenidos para todas las variables evaluadas (PG, TMG, VMG y E) fueron obtenidos a una temperatura constante de $35^{\circ} \mathrm{C}$ pudiéndose considerar esta, como la temperatura óptima de germinación. 


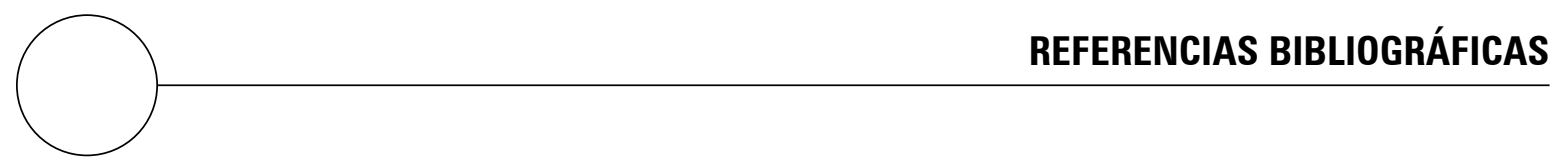

Armitage, A.M. 1994. Ornamental bedding plants. CAB International, Wallingford, UK.

Ba, O., M. Sagna y M. Ourèye. 2012. Germination capacity of annonaceae seeds (Annona muricata L., A. squamosa L. and A. senegalensis Pers.) cultivated under axenic conditions. Int. J. Sci. Adv. Technol. $2(6), 27-33$

Baskin, C.C. y J.M. Baskin. 2001. Seeds. Ecology, biogeography, and evolution of dormancy and germination. Academic Press, San Diego, CA.

Bewley, J.D. y M. Black. 1994. Seeds: Physiology of development and germination. Plenum Press, New York, NY.

Bewley, J.D. y M. Black. 1982. Physiology and biochemistry of seeds in relation to germination. Vol. II. Viability, dormancy and environmental control. Springer-Verlag, Berlin.

Borges, E. y A.B. Rena. 1993. Germinação de sementes. pp. 83-135. En: Aguiar, I., F.C.M. Pinã-Rodrigues y M.B. Figlioglia (eds.). Sementes florestais tropicais. Abrates, Brasília.

Carvalho, N. y J. Nakagawa. 2000. Sementes. Ciencia, tecnología e producao. Funep, Jaboticabal, Brasil.

Castro, M. 2003. Influência de diferentes temperaturas na germinação de sementes de Tabebuia capitata (Bur. et K. Schum. Sandw.) Trabajo de grado. Instituto de Tecnologia de la Amazonía, Universidad de Manaus, Manaus, Brasil.

Cavalcante, A. y S. Pérez. 1995. Efeitos da temperatura sobre a germinacao de sementes de Leucaena leucocephala (LAM) de WIT. Rev. Bras. Sementes 17(1), 1-8.

Come, D. 1982. Germination, pp. 129-225. En: P. Mazliak (ed.). Croissance et développement, Physiologie végétale II. Hermann, Paris.

Ferreira, G., E. Cereda, C. Silva, R.J.P. Cunha y A. Cataneo. 1997. Imbibition studies of sugar apple (Annona squamosa L.) and atemoya (Annona Hibrid) seed. pp. 210-225. En: Memorias II Congreso International de Anonáceas. Universidad Autonoma Chapingo, Chapingo, México.

George, A.P. 1985. Custard apple. pp. 3-41. En: O. Cairns (ed.). Tropical tree fruits for AUS. Queensland Department of Primary Industries, Brisbane, Australia.
George, A. y R.J. Niessen. 1987. Propagation of Annona species: a review. Sci. Hortic. 33, 75-85.

George. A.P. y R. Nissen, 1993. Annonaceous. pp. 198199. En: Macrae, R., R.K. Robinson y M.I. Sadla (eds.). Encyclopedia of food science, food technology and nutrition. Academic Press, London.

Godoi, S. y M. Takaki. 2004. Effects of light and temperature on seed germination in Cecropia hololeuca Miq. (Cecropiaceae). Braz. Arch. Biol. Technol. 47(2), 185-191.

Hartmann, H.T., D.E. Kester y F.T. Davies. 1997. Plant propagation: Principles and practices. Prentice Hall International, New Jersey, N.J.

Harty, R.L. y J. Butler. 1975. Temperature requirements for germination of green panic, Panicum maximum var. trichoglume, during the after-ripening period. Seed Sci. Technol. 3(2), 529-536.

Hoyos, J. 1989. Frutales en Venezuela. Sociedad de Ciencias Naturales La Salle, Caracas.

Kavati, R. 1992. Cultivo da atemóia. pp. 39-70. En: Donadio, L., A. Martins y J. Valente (eds.). Fruticultura tropical. Funep, Jaboticabal, Brasil.

Lobo, M., Ó. Delgado, J. Cartagena, E. Fernández y C. Medina. 2007. Categorización de la germinación y la latencia en semillas de chirimoya (Annona cherimola L.) y guanábana (Annona muricata L.), como apoyo a programas de conservación de germoplasma. Agron. Colomb. 25(2), 231-244.

Lotero, A. 1976. Géneros de la flora de Colombia III, "Anonaceae". Trabajo de grado. Universidad Nacional de Colombia, Bogotá.

Martínez, F. 2012. Caracterización morfoanatómica de semillas de anón (Annona squamosa L.) y evaluación de algunos parámetros fisiológicos del proceso de germinación y latencia. Tesis de maestría. Facultad de Agronomía, Universidad Nacional de Colombia, Bogotá.

Mayer, AM. y A. Poljakoff-Mayber. 1989. The germination of seeds. Pergamon Press. Oxford. 270 p.

Morton, J. 1987. Sugar apple (Annona squamosa). pp. 69 72. En: Morton, J. (ed.). Fruits of warm climates. Creative Resources Systems, Miami, FL.

Moreno, R., L. Luna y R. Gonzalez. 1999. Estudios sobre la germinación de Annona lutescens. p. 82. En 
Memorias II Congreso Internacional de Anonáceas. Universidad de Ciencias y Artes del Estado de Chiapas, Tuxtla Gutiérrez, Chiapas, México.

Nepomuceno Costa, P., S. Bueno y G. Ferreira. 2011. Fases da germinação de sementes de Annona emarginata (Schltdl.) H. Rainer em diferentes temperaturas. Rev. Bras. Frutic. 33(1), 253-260.

Oliveira, I., R. Andrade y A. Martins. 2005. Influência da temperatura na germinação de sementes de Annona montana. Rev. Bras. Frutic. 27(2), 344-345.

Padilla, I. y C.L. Encina. 2003. In vitro germination of cherimoya (Annona cherimola) seeds. Sci. Hortic. 97, 219-227.

Popenoe, W. 1974. The Annonaceous fruits. pp. 161-195. En: W. Popenoe (ed.). Manual of tropical and subtropical fruits. Hafner Press, New York, NY.

Pinto, A.C. y E.M. Silva. 1975. Influencia de hormono sobre o podor germinativo de sementes de graviola (Annona muricata L.). pp. 415-421. En: Anais do III Congresso Brasileiro de Fruticultura, V. II. Sociedade Brasileira do Fruticultura, Rio de Janeiro, Brasil.

Pinto, A.C. 2005a. Chapter 3: Origin and distribution. pp. 17-20. En: Williams, J.T., R.W. Smith, A. Hughes, N. Haq y C.R. Clements (eds.). Annona species. International Centre for Underutilized Crops, University of Southampton, Southampton, UK.

Pinto, A.C. 2005b. Chapter 10. Agronomy. pp. 70-123. En: Williams, J.T., R.W. Smith, A. Hughes, N. Haq y C.R. Clements. (eds.). Annona species. Interna- tional Centre for Underutilized Crops, University of Southampton, Southampton, UK.

Ranal, M. y D.G. Santana. 2006. How and why to measure the germination process? Rev. Brasil. Bot. 29(1), 1-11.

Reino, J., Y. González y J. Sánchez. 2008. Optimum germination temperature and imbibition patterns of the seeds from Albizia lebbeck, Gliricidia sepium and Bauhinia purpurea. Pastos Forrajes 31(3), 209-216.

Toll-Jubes, J., H. Martínez, E. Padilla y C. Oeste. 1975. Efectos de escarificación, medio, posición de siembra y ácido gibberéllico, sobre la germinación de semillas en chirimoya (Annona cherimolia Mill.). Rev. Agron. Argent. 12(1-2), 161-172.

Tybirk, K. 1991. Régénération des légumineuses ligneuses du Sahel. Aarhus Report. 28. Botanical Institute, Aarhus University, Dinamarca.

Van Vuuren, J. y A. Lubbe. 2000. The influence of temperature on the germination of seed of a commercial verbena hybrid. Acta Hort. 515, 185-192.

Wagner, J.A., R.S. Alexandre, J.R. da Silva, L. Duarte, J.O. da Costa y C.H. Bruckner. 2006. Influência do substrato na germinação e desenvolvimento inicial de plantas de maracujazeiro amarelo (Passiflora edulis Sims f. flavicarpa Deg). Ciênc. Agrotec. 30(4), 643-647.

Zucareli, V., G. Ferreira, E. Silvério y A. Amaro. 2007. Luz e temperatura na germinação de sementes de Annona squamosa L. Rev. Bras. Biociências 5(2), 840-842. 